wissen kompakt 2017 $11: 1-2$

DOI 10.1007/s11838-016-0038-3

Online publiziert: 11. Januar 2017

(c) Springer Medizin Verlag Berlin und Freier

Verband deutscher Zahnärzte e.V. 2017

CrossMark

\section{H. Dommisch}

Abteilung für Parodontologie und Synoptische Zahnmedizin, CharitéCentrum für Zahn-, Mund- und Kieferheilkunde, Charité - Universitätsmedizin Berlin, Berlin, Deutschland

\section{Dentale Traumatologie}

\section{Aspekte aus Klinik und Wissenschaft}

Die Traumatologie stellt ein sehr komplexes Teilgebiet der Zahnmedizin dar und bringt vielzählige Herausforderungen mit sich. Diese können sowohl patienten-, situations- als auch zahnbedingter Art sein. Patientenbezogene Faktoren betreffen v. a. das Alter der Patienten. Oftmals sind die betroffenen Patienten Kinder und Jugendliche, da die Inzidenz in dieser Bevölkerungsgruppe aufgrund von Stürzen, körperbetonten Sportarten und der während des Durchbruchs sehr exponierten Zähne vergleichsweise hoch ist. Die Therapie von Traumata bei diesen Patienten erfordert neben dem Fachwissen auch viel Geduld und Einfühlungsvermögen vonseiten des behandelnden ärztlichen/zahnärztlichen Teams. Die sich im Wachstum befindlichen Patienten müssen oft mit einer langwierigen Therapie rechnen, weshalb die Compliance eine entscheidende Rolle spielt.

Situationsbezogene Faktoren sind oftmals weitere Verletzungen, die vielfach zunächst eine chirurgische Sofortversorgung erfordern. Diese kann zwar den Beginn der Therapie der Zahntraumata verzögern, ist aber gleichzeitig oft ein entscheidender Schritt für den weiteren Heilungsverlauf und damit auch die Prognose der beteiligten Zähne. Hier ist eine zeitnahe und optimale interdisziplinäre Kooperation zwischen den einzelnen behandelnden Kollegen erforderlich, um die Therapie für die in der Regel sehr jungen Patientinnen und Patienten so sinnvoll wie möglich zu planen und $\mathrm{zu}$ terminieren. Zahnbezogene Faktoren betreffen das Ausmaß der Schädigung von Zähnen und Parodont und bestimmen maßgeblich Therapie und Prognose. Die Art der Verletzung entscheidet demnach auch über die zu beteiligenden Fachrich- tungen, mögliche zukünftige Komplikationen sowie das optimale Ergebnis der geplanten Therapie.

\section{》) Die Behandlung dentaler \\ Traumata erfordert eine optimale interdisziplinäre Kooperation}

Die Therapie von Traumata der Zähne und des Zahnhalteapparats erfordert demnach die patientenorientierte Zusammenarbeit von verschiedenen Disziplinen der Zahnmedizin wie der zahnärztlichen Chirurgie, der Zahnerhaltung (speziell der Endodontologie), der Parodontologie, der Prothetik und der Kieferorthopädie. Am Anfang sollten immer eine ausführliche Anamneseerhebung und Befundung stehen, um anhand der daraus resultierenden Diagnosen eine interdisziplinäre Priorisierung und Planung vornehmen zu können. Die Anamnese ermöglicht es, den Unfallhergang genau $\mathrm{zu}$ rekonstruieren, die Dauer zwischen Unfall und Erster Hilfe, die Dauer der extraoralen Verweilzeit avulsierter Zähne sowie Risikopatienten zu identifizieren. Im Rahmen der Befundaufnahme wird das gesamte Ausmaß der Traumatisierung dokumentiert, indem die Hart- und Weichgewebe präzise inspiziert werden. Zusammen mit der Anamnese trägt die intensive Befundaufnahme erheblich zu einer optimalen Erfassung der klinischen Situation und damit zur realistischen Einschätzung der individuellen Prognose der jungen Patienten bei.

Die Prävalenz der dentalen Traumata ist v. a. bei den 7- bis 12-jährigen Kindern bzw. Jugendlichen am höchsten. Besonders häufig sind hierbei die bleibenden Frontzähne betroffen, welche sich oft im Rahmen des Zahnwechsels noch im Durchbruch befinden. Das Lebensalter der betroffenen Patienten ist hinsichtlich des Körperwachstums besonders entscheidend. In Hinblick auf das Kieferwachstum ist der physiologische Zahnwechsel, die Zähne selbst im Alveolarknochen und die Entzündungsfreiheit der dentalen sowie parodontalen Strukturen entscheidend. Daher ist es unbedingtes Ziel, die traumatisierten Weich- und Hartgewebsstrukturen, so es möglich ist, zu erhalten.

Das Spektrum der dentalen Traumata umfasst jedoch unkomplizierte Schmelz- bzw. Schmelz-Dentin-Frakturen, komplizierte Kronenfrakturen mit unterschiedlichem Ausmaß, Intrusionen, Extrusionen, laterale Dislokationen und Avulsionen, welche oftmals mit einer Fraktur der alveolären Knochenstrukturen einhergehen. Besonders bei den letzteren Arten dentaler Traumata werden neben den Hartgeweben ebenfalls die parodontalen Weichgewebe wie das Desmodont und die Gingiva sowie das Gewebe der Zahnpulpa erheblich geschädigt. Je nach Ausmaß des Traumas kann es eine Herausforderung sein, den Schweregrad der desmodontalen und endodontalen Schädigung zu erfassen.

Durch moderne Therapieverfahren in der zahnärztlichen Chirurgie, der Endodontologie, der Kieferorthopädie und der restaurativen Zahnmedizin können teilweise erheblich kompromittierte Weichund Hartgewebe erhalten bzw. gegebenenfalls restauriert werden. Besonders in der Endodontologie konnten in den vergangenen Jahren bedeutende Fortschritte verzeichnet werden. So können heute Zähne mit noch nicht vollständig abgeschlossenem Wurzelwachstum bei gegebener Indikation revitalisiert 
werden, wenn diese aufgrund eines dentalen Traumas nekrotisches Zahnpulpagewebe aufweisen. Besonders im Wechselgebiss ergeben sich zahlreiche Herausforderungen für die endodontische und restaurative Therapie. Allein die Applikation des Kofferdams für die sterile endodontische Therapie sowie für die blut- und speichelfreie restaurative Versorgung der Zahnhartsubstanzen kann zuweilen aufgrund der im Durchbruch befindlichen Zähne besonders erschwert sein.

Übergeordnetes Ziel ist es, die Entzündung parodontaler und endodontaler Gewebe zu kontrollieren und die Funktionalität der Zahnstrukturen wiederherzustellen. Die Entzündung ist nach einem dentalen Trauma entscheidend, denn diese kann, je nach Art der Verletzung, zu Resorptionen der Hartsubstanzen der Zahnwurzel führen. Die traumatische Schädigung der desmodontalen Zellen und Fasern sowie des Zahnzements bestimmen, ob resorptive Prozesse einsetzen. Zusätzlich kann eine endodontische Infektion den Prozess der Resorption beschleunigen. Die entzündliche Resorption und die Ersatzresorption sind hierbei häufige Phänomene. Beide Arten beeinflussen die Prognose entscheidend und führen zu weitreichenden Problemen hinsichtlich des physiologischen Kieferwachstums. Daher sollten sich die einzelnen spezialisierten Fachbereiche gegenseitig konsultieren und ggf. gemeinsam agieren, um für die zumeist jungen $\mathrm{Pa}$ tienten Entzündungsfreiheit und eine optimale Rehabilitation der Weich- und Hartgewebsstrukturen zu ermöglichen.

\section{》) Ziel ist es, die Weich- und Hartgewebsstrukturen zu erhalten}

Diese Ausgabe von wissen kompakt befasst sich eingehend mit der Problematik dentaler Traumata. Die einzelnen Kapitel diskutieren die aktuellen Aspekte der zahnärztlichen Traumatologie in Hinblick auf die chirurgische Therapie, neue wissenschaftliche Erkenntnisse sowie das klinische Prozedere im Rahmen der endodontischen Revitalisierung und das aktuelle Wissen zur modernen restaurativen Behandlung von Kronenfrakturen.

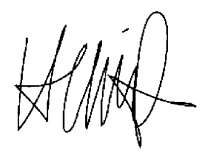

Prof. Dr. H. Dommisch

\section{Korrespondenzadresse}

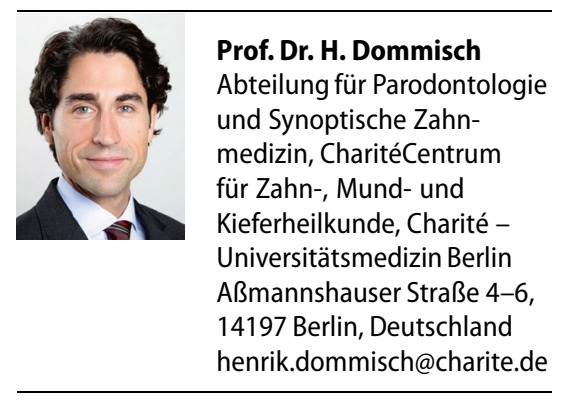

Interessenkonflikt. H. Dommisch gibt an, dass kein Interessenkonflikt besteht. 